\title{
Finite temperature studies of Te adsorption on $\mathrm{Si}(001)$
}

\author{
Prasenjit Sen ${ }^{\text {a }}$, S. Ciraci ${ }^{\text {a,b }}$, Inder P. Batra ${ }^{\text {a,* }}$, C.H. Grein ${ }^{\text {a }}$, S. Sivananthan ${ }^{\text {a }}$ \\ ${ }^{a}$ Department of Physics (MC273), University of Illinois at Chicago, 845 West Taylor Street, Chicago, IL 60607-7059, USA \\ b Department of Physics, Bilkent University, Bilkent, Ankara 06533, Turkey \\ Received 11 January 2002; accepted for publication 1 August 2002
}

\begin{abstract}
We perform first principles density functional calculations to investigate the adsorption of $\mathrm{Te}$ on the $\mathrm{Si}\left(\begin{array}{ll}0 & 0\end{array}\right)$ surface from low coverage up to a monolayer coverage. At low coverage, a Te atom is adsorbed on top of the Si surface dimer bond. At higher coverages, Te atoms adsorption causes the $\mathrm{Si}-\mathrm{Si}$ dimer bond to break, lifting the $(2 \times 1)$ reconstruction. We find no evidence of the Te-Te dimer bond formation as a possible source of the $(2 \times 1)$ reconstruction at a monolayer coverage. Finite temperature ab initio molecular dynamics calculations show that Te covered $\operatorname{Si}(001)$ surfaces do not have any definitive reconstruction. Vibrations of the bridged Te atoms in the strongly anharmonic potentials prevent the reconstruction structure from attaining any permanent, two-dimensional periodic geometry. This explains why experiments attempting to find a definite model for the reconstruction reached conflicting conclusions. (c) 2002 Elsevier Science B.V. All rights reserved.
\end{abstract}

Keywords: Density functional calculations; Molecular dynamics; Adatoms; Growth; Silicon; Surface relaxation and reconstruction

\section{Introduction}

Adsorption of Te on the $\operatorname{Si}(001)$ surface is of great relevance to various technological applications including its role as a surfactant in epitaxial growth. While the objective in the homoepitaxial growth of $\mathrm{Si}$ on the $\mathrm{Si}\left(\begin{array}{lll}0 & 0 & 1\end{array}\right)$ surface is growth with the least possible number of defects, the relatively large diffusion length of $\mathrm{Si}$ adatoms on the surface does not permit this objective to be achieved. The growth usually takes place in the Stranski-Kras-

\footnotetext{
${ }^{*}$ Corresponding author. Tel.: +1-312-413-2798; fax: +1-312996-9016.

E-mail address: ipbatra@uic.edu (I.P. Batra).
}

tanov mode, where three-dimensional (3D) islands form. Similarly, the epitaxial growth of Ge on the $\operatorname{Si}\left(\begin{array}{lll}0 & 0 & 1\end{array}\right)$ surface falls in the same category, where, after the first few layers of $\mathrm{Ge}$, the subsequent growth is in the form of $3 \mathrm{D}$ islands. However, thick layers of Ge can be grown layer-by-layer on Si by introducing a monolayer or submonolayer of a surfactant like As [1-3], Sb [4,5] or Te [6-8]. Such layer-by-layer homoepitaxial growth of Si crystals can also be achieved by introducing Te surfactants on the $\operatorname{Si}\left(\begin{array}{ll}0 & 1\end{array}\right)$ surface. Te is also known to be an excellent surfactant in the growth of $\operatorname{GaAs}\left(\begin{array}{lll}0 & 0 & 1\end{array}\right)$ [14]. However, the detailed surfactant mechanism is not understood in either situation [9-13].

The goal of combining large infra-red detector arrays with relatively cheap and well developed $\mathrm{Si}$ 
integrated circuit read out technology has led to efforts to grow $\mathrm{HgCdTe}$ (MCT) on $\mathrm{Si}\left(\begin{array}{l}0 \\ 0\end{array} 1\right)$. Because of a large $(\sim 19 \%)$ lattice mismatch between $\mathrm{Si}$ and MCT, thick buffer layers of CdTe are often grown prior to depositing active layers of MCT. MCT is the actual infra-red detector material. It has been observed [15] that the growth of CdTe layers on the $\operatorname{Si}\left(\begin{array}{lll}0 & 0 & 1\end{array}\right)$ surface commences with an initial monolayer or submonolayer of Te. An understanding of the atomic configuration of adsorbed Te, which forms the first grown layer on the bare $\operatorname{Si}\left(\begin{array}{ll}0 & 0\end{array}\right)$ surface, is crucial for the fabrication of high performance devices since it determines how growth nucleates and hence the quality of the epilayer [16]. Because of the immense technological applications of these materials, it becomes important to understand the reconstruction of the $\mathrm{Si}\left(\begin{array}{lll}0 & 0 & 1\end{array}\right)$ surface with a Te ad-layer.

Recently a number of experiments [15,17-21,24] have investigated the structural transition of the $\mathrm{Si}(001)-(2 \times 1)$ surface due to Te adsorption. But there have been only a few theoretical studies $[25,26]$. In particular, the issues that need to be understood are the relative energetics of Te adatoms at low coverages at various sites on the $\mathrm{Si}\left(\begin{array}{lll}0 & 0 & 1\end{array}\right)-(2 \times 1)$ surface, breaking of the $\mathrm{Si}-\mathrm{Si}$ dimers with increasing Te coverage and the possibility of Te-Te dimer formation at nearly $1 \mathrm{ML}$ coverage, and the geometry of the surface with 1 ML of Te. This last point has generated some debate. While most of the experiments conclude that at $1 \mathrm{ML}, \mathrm{Te}$ atoms break the Si dimer bonds and lead to a $(1 \times 1)$ surface, Tamiya et al. [24] claim that though the surface is $(1 \times 1)$ at low temperatures, at around $600{ }^{\circ} \mathrm{C}$ the Te adatoms undergo rearrangement and the surface becomes $(2 \times 1)$. Wiame et al. [21] interpreted their STM data in terms of a $(2 \times 1)$ surface reconstruction and the formation of asymmetric Te-Te dimers. The Te adsorbed GaAs( 001$)$ surface is known to show such variable reconstruction with changing temperature $[22,23]$. The differing reconstruction geometries deduced from different experiments present a puzzling situation.

In this work, we make substantial efforts to resolve these issues by performing ab initio density functional calculations. Particular atomic structures with well-defined reconstruction geometries are verified by performing extensive total energy calculations at $T=0 \mathrm{~K}$ and geometry optimizations. Atomic configurations corresponding to the global or local minima on a Born-Oppenheimer surface are then attributed to stable surface structures. We also perform finite temperature molecular dynamics (MD) calculations to access different reaction pathways that can lead to possible reconstruction patterns and hence to address the question of a $(2 \times 1)$ surface at elevated temperatures, as reported in Ref. [24].

We find that the on-top position above the dimer bond on the $\operatorname{Si}\left(\begin{array}{lll}0 & 0 & 1\end{array}\right)$ surface is energetically the most favorable for a Te ad-atom. At a coverage between $0.5<\Theta \leqslant 1$, where $\Theta$ is the fractional coverage of the Si surface (i.e., the number of Te atoms per surface $\mathrm{Si}$ atom), the $\mathrm{Si}-\mathrm{Si}$ dimer bonds are broken and $\mathrm{Si}$ atoms are pushed to their ideal bulk positions. The Te-Te dimer formation is found to be energetically unfavorable in our calculations. There is also no indication of a $(2 \times 1)$ surface reconstruction at any temperature. The energy barrier for the Te atoms to move from their optimal positions is very small for displacements of up to $\sim \pm 0.5 \AA$. Uncorrelated lateral excursions of Te atoms bridged to surface silicon atoms, in this flat potential well, prevent them from attaining any definitive surface reconstruction pattern.

The organization of the rest of the paper is as follows. In Section 2, we briefly describe the method and the relevant parameters of our calculations. Results of our extensive total energy calculations at $T=0 \mathrm{~K}$ and geometry optimizations for the clean and the Te adsorbed $\operatorname{Si}\left(\begin{array}{lll}0 & 0 & 1\end{array}\right)$ surface at very low coverage are presented in Section 3. Here, we have calculated binding energies of Te adatoms at $\Theta=0.0625$ at four special symmetry positions of the $\operatorname{Si}(001)$ surface and have studied the behavior of $\mathrm{Si}-\mathrm{Si}$ dimer bonds on the reconstructed surface with increasing uniform Te coverage $0.0625<\Theta<0.5$. In Section 4 , we investigate the breaking of the $\mathrm{Si}-\mathrm{Si}$ dimer bond by the adsorbed Te atom, and address the issue as to whether the adsorbed Te atoms form Te-Te dimer bonds leading to a $(2 \times 1)$ surface reconstruction. In Section 5, we discuss ab initio MD calculations performed at 600 and $1000 \mathrm{~K}$, and analyze the nature of atomic displacements at finite tempera- 
ture which reveal the physics underlying the unusually long excursions of the Te atoms. We also discuss the implications of these uncorrelated displacements in the observed reconstruction patterns. Our key results are summarized in Section 6 . Some of our results were reported briefly recently [27].

\section{Method}

Calculations were carried out within density functional theory. The wave functions are expressed by plane waves with the cutoff energy $|k+G|^{2} \leqslant 250 \mathrm{eV}$. The Brillouin zone (BZ) integrations are performed by using the MonkhorstPack scheme [28] with $(2 \times 2 \times 1),(2 \times 8 \times 1)$ and $(4 \times 8 \times 1) k$-point meshes for $(4 \times 4),(4 \times 1)$ and $(2 \times 1)$ cells, respectively. The convergence with respect to the energy cutoff and number of $k$ points was tested. Ionic potentials are represented by ultra-soft Vanderbilt type pseudopotentials [29] and results for the fully relaxed atomic structures are obtained using the generalized gradient approximation (GGA) [30]. The preconditioned conjugate gradient method is used for wave function optimization and conjugate gradient method for ionic relaxation at $T=0 \mathrm{~K}$. At finite temperatures, the Nosé-Hoover thermostat [31] is employed for constant temperature $\mathrm{MD}$ of ionic motions in the self-consistent field of the electrons $[32,33]$. The time step in MD calculations, $\Delta t$, is chosen such that a typical phonon time period is divided into a few tens of time steps. We picked $\Delta t$ to be $2 \mathrm{fs}$ to ensure that the ionic trajectories are smooth. The $\operatorname{Si}(001)$ surface is represented by a repeated slab geometry. Each slab contains $5 \mathrm{Si}$ atomic planes with hydrogen atoms passivating the $\mathrm{Si}$ atoms at the bottom of the slab. Consecutive slabs are separated by a vacuum space of $9 \AA$ A. For calculations at $T=0 \mathrm{~K}, \mathrm{Si}$ atoms in the top four atomic layers are allowed to relax, while the bottom $\mathrm{Si}$ atoms and passivating hydrogens are fixed to simulate bulk-like termination.

In finite temperature calculations, all atoms, including $\mathrm{H}$ atoms at the bottom, are allowed to move to avoid a large temperature gradient. Lattice parameters are expanded according to the temperature under study using the experimental thermal expansion coefficient in order to prevent the lattice from experiencing internal thermal strain. We reproduced the energetics and geometry of the $\mathrm{c}(4 \times 2), \mathrm{p}(2 \times 2)$ and $\mathrm{p}(2 \times 1)$ reconstructions of a clean $\operatorname{Si}(001)$ surface using the above parameters [34].

\section{Low Te coverage}

In order to test the parameters described in Section 2, we calculated the total energies of the $\mathrm{c}(4 \times 2), \mathrm{p}(2 \times 2)$ and $\mathrm{p}(2 \times 1)$ reconstructions of the clean $\mathrm{Si}\left(\begin{array}{ll}0 & 0\end{array}\right)$ surface in a supercell comprising a $(4 \times 4)$ ideal cell (composed of $80 \mathrm{Si}$ atoms and $32 \mathrm{H}$ atoms). The $\mathrm{Si}$ atoms in the top four layers are relaxed for the optimization of the atomic structure. Our results are in good agreement with the earlier density functional calculations [34]. For the $\mathrm{p}(2 \times 1)$ surface with asymmetric $\mathrm{Si}-\mathrm{Si}$ dimers, there is an energy gain of $1.6 \mathrm{eV}$ per dimer. The $\mathrm{Si}-$ Si dimer distance is $2.30 \AA$ and the dimer bond makes an angle of $18^{\circ}$ with the horizontal plane. The optimized atomic positions are given in Table 1 . Though the $\mathrm{p}(2 \times 2)$ and $\mathrm{c}(4 \times 2)$ surfaces have slightly lower energies, the energy differences with the $\mathrm{p}(2 \times 1)$ surface are small, and we work with the latter reconstruction for further adsorption studies.

Table 1

Ideal bulk terminated atomic positions (in $\AA$ ) for the top four layers of $\operatorname{Si}(001)$ in a $2 \times 1$ supercell and the displacement of each atom in reaching the optimized $2 \times 1$ reconstructed surface

\begin{tabular}{cllllrr}
\hline \multirow{2}{*}{$\mathrm{N}$} & \multicolumn{2}{l}{ Ideal lattice } & & \multicolumn{2}{c}{ Optimized } \\
\cline { 2 - 4 } & $x$ & $y$ & $z$ & & \multicolumn{1}{c}{$\Delta x$} & \multicolumn{1}{c}{$\Delta z$} \\
\hline 1 & 0.0 & 0.0 & 6.30 & & 1.15 & -0.60 \\
2 & 3.84 & 0.0 & 6.30 & & -0.51 & 0.14 \\
3 & 0.0 & 1.92 & 4.94 & & 0.06 & 0.01 \\
4 & 3.84 & 1.92 & 4.94 & & -0.13 & 0.11 \\
5 & 1.92 & 1.92 & 3.58 & & 0.05 & -0.10 \\
6 & 5.76 & 1.92 & 3.58 & & -0.06 & 0.12 \\
7 & 1.92 & 0.0 & 2.23 & & 0.0 & -0.08 \\
8 & 5.76 & 0.0 & 2.23 & & -0.01 & 0.06 \\
\hline
\end{tabular}

The $y$ coordinates do not change for this reconstruction and hence $\Delta y$ 's are all zero. 

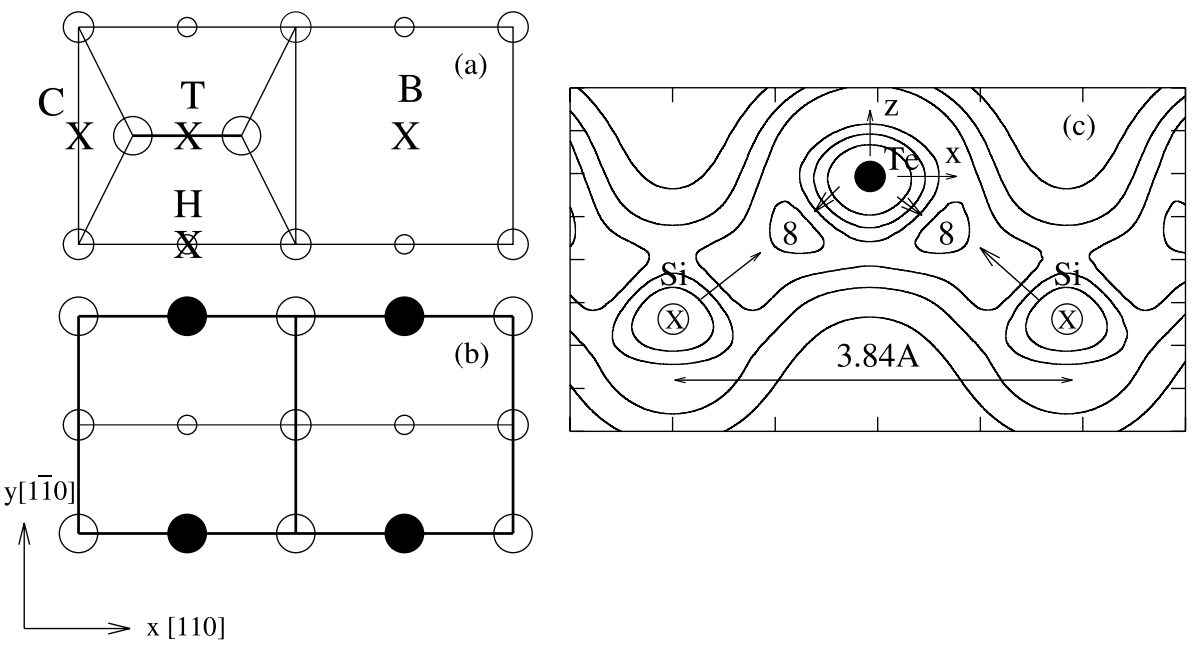

Fig. 1. (a) The unit cell of the $\operatorname{Si}\left(\begin{array}{lll}0 & 0 & 1\end{array}\right)-(2 \times 1)$ surface. The possible sites for the adsorption of Te at very low $\Theta$ are marked by $\mathbf{X}$. Large empty, small empty, and smallest empty circles denote first layer $\mathrm{Si}$, second layer $\mathrm{Si}$, and third layer $\mathrm{Si}$ atoms, respectively. The lines between circles indicate bonds. The surface dimer bond is shown by a thicker line. (b) Ideal $(1 \times 1) \mathrm{Te}$ covered $\mathrm{Si}(001)$ surface at $\Theta=1$. The filled circles denote the Te atoms. (c) Charge density contours on the $\mathrm{Si}-\mathrm{Te}-\mathrm{Si}$ plane for the structure shown in (b). There is a small charge transfer from the $\mathrm{Si}$ to the Te atoms.

We calculated the binding energies of a single Te adsorbed on the four symmetry sites of the $\mathrm{Si}\left(\begin{array}{lll}0 & 0 & 1\end{array}\right)-(2 \times 1)$ unit cell, i.e. on-top $\mathrm{T}$, cave $\mathrm{C}$, hollow H, and bridge B sites shown in Fig. 1(a). Calculations were performed using a large square supercell of sides $15.36 \AA$ consisting of eight $(2 \times 1)$ cells, laid out in a $(2 \times 4)$ configuration. The large size of the supercell ensures that the interaction between an adsorbed Te atom and its periodic images is negligible so that results represent an "isolated" Te $(\Theta=0.0625)$. In Fig. 1, only one $(2 \times 1)$ cell of the supercell is shown. The binding energies, $E_{\mathrm{b}}$, are defined as

$E_{\mathrm{b}}=E_{\mathrm{T}}[\mathrm{Si}]+E_{\mathrm{a}}[\mathrm{Te}]-E_{\mathrm{T}}[\mathrm{Si}+\mathrm{Te}]$,

where $E_{\mathrm{T}}[\mathrm{Si}]$ is the total energy of the $\mathrm{Si}$ slab; $E_{\mathrm{T}}[\mathrm{Si}+\mathrm{Te}]$ is the total energy with a Te adsorbed on it, and $E_{\mathrm{a}}[\mathrm{Te}]$ is the energy of a single isolated Te atom. The total energies of the slab with and without $\mathrm{Te}$ are calculated in the same supercell with fully optimized atomic configuration at $T=0$ $\mathrm{K}$. The binding energies are found to be $\mathrm{T}: 4.5 \mathrm{eV}$, C: $3.5 \mathrm{eV}, \mathrm{H}: 3.4 \mathrm{eV}$, and B: $3.2 \mathrm{eV}$. The most energetic site at low coverage is the $\mathrm{T}$ site, where the Te atom adsorbs above the middle of the dimer bond of the $\operatorname{Si}\left(\begin{array}{ll}0 & 01\end{array}\right)-(2 \times 1)$ surface and is bonded to the two $\mathrm{Si}$ atoms forming the dimer. This is consistent with our intuitive chemical notion that $\mathrm{Te}\left(5 \mathrm{p}^{4}\right)$ tries to fill its outermost $p$-shell by coordinating with two surface $\mathrm{Si}$ atoms. This way the dangling bonds of the surface Si atoms are also saturated. Our results are consistent with STM experiments [17] and are also in agreement with two earlier calculations [25,26]. Miwa et al. calculated the binding energies for an isolated Te at five different sites on the $\operatorname{Si}(001)-(2 \times 1)$ surface. The "bridge", "vacancy" and "cave-a" sites in their paper are the same as T, H and B sites here. We find the $\mathrm{H}$ and $\mathrm{B}$ sites to have very similar binding energies, though the $\mathrm{H}$ site energy is a bit more favorable. The $\mathrm{T}$ site binding energy is $1.3 \mathrm{eV}$ higher than the B site binding energy. They also find the "bridge" (T) site to be the most favorable one for a Te with a binding energy $0.7 \mathrm{eV}$ higher than that at the "cave-a" (B) site. Like us, their calculations also find "vacancy" $(\mathrm{H})$ and "cave-a" (B) sites to have very similar binding energies with the "cave-a" (B) site having marginally higher binding energy. Takeuchi, on the other hand, considers the T and B sites (note that the "cave" site in his paper is our B site) and finds the T site to have higher binding energy by about $0.8 \mathrm{eV}$. Thus 
all the calculations reach the same conclusion, that the top of the Si dimer bond is the most favorable site for adsorption of an isolated Te. There are some differences in the binding energy values and that may be due to different system sizes used in various calculations. For example, Miwa et al. used a $(2 \times 2)$ surface supercell while we have a $(4 \times 4)$ supercell for these calculations.

We examined the stability of the adsorbed Te atom and the underlying $\mathrm{Si}-\mathrm{Si}$ dimer at higher coverages. At $\Theta=0.5$, we placed one Te adatom on top of each $\mathrm{Si}-\mathrm{Si}$ dimer bond in a $(2 \times 1)$ unit cell. Upon optimizing the structure, the dimer bonds are only slightly weakened but not broken. The $\mathrm{Si}-\mathrm{Si}$ dimer distance increases marginally to $2.31 \AA$ from the clean surface value of $2.30 \AA$. But more importantly, Te atoms remove the asymmetry between the two $\mathrm{Si}$ atoms in the dimer. The height of the adsorbed Te atoms is $2.25 \AA$ above the top Si layer, and the calculated $\mathrm{Si}-\mathrm{Te}$ distance of $2.53 \AA$ is in good agreement with experiment [20]. This value is also close to the sum of covalent radii of $\mathrm{Si}(1.17 \AA)$ and $\mathrm{Te}(1.32 \AA)$. However, we do not see any 'ripple' in the Te overlayer at $\Theta=0.5$ or at any other coverage, as reported in the Ref. [20].

\section{Te coverage up to one monolayer}

For $\Theta>0.5$, Te atoms must start occupying other less favorable sites since all the on-top dimer sites are already occupied at $\Theta=0.5$. We begin with an initial configuration where one $\mathrm{Te}$ is adsorbed at the $\mathrm{T}$ site and the second one at the $\mathrm{B}$ site on the $\operatorname{Si}(001)-(2 \times 1)$ surface corresponding to $\Theta=1$, and optimize this structure at $T=0 \mathrm{~K}$. The occupation of the $\mathrm{B}$ site at high Te coverage is consistent with experiments [15], even though the $\mathrm{B}$ site is not the second most bound site at low coverages. The energetics is expected to change when two Te atoms are adjacent to each other due to adsorbate induced interactions. In reaching the stable structure, the $\mathrm{Te}$ atoms form directional bonds with surface $\mathrm{Si}$ atoms while $\mathrm{Si}-\mathrm{Si}$ dimer bonds elongate and eventually break. Each $\mathrm{Si}-\mathrm{Si}$ dimer bond is broken to form four new $\mathrm{Si}-\mathrm{Te}$ bonds. In the final stable structure, $\mathrm{Si}$ atoms of the broken dimer bond are pushed to their bulk positions, reforming the outermost, bulk-like $\operatorname{Si}\left(\begin{array}{lll}0 & 0 & 1\end{array}\right)$ atomic plane. Each adsorbed Te atom is connected to the substrate by two Te-Si bonds of length 2.53 A. In the end, a $\operatorname{Te}\left(\begin{array}{lll}0 & 0 & 1\end{array}\right)$ atomic plane forms 1.65 $\AA$ above the Si substrate with a binding energy of $4.28 \mathrm{eV}$ per Te atom. These values for the $\mathrm{Te}-\mathrm{Si}$ bond length, and the height of the Te layer from the $\mathrm{Si}$ substrate are in very good agreement with the surface extended X-ray absorption fine structure (SEXAFS) and X-ray standing wave (XSW) spectroscopy experiments of Burgess et al. [20] They find a Te-Si bond length of $2.52 \pm 0.05 \AA$ and a Te layer height of $1.65 \pm 0.03 \AA$. At a monolayer coverage, our calculations are also in agreement with the results reported by Miwa et al [25]. Fig. 1(b) shows the atomic arrangement of this ideal $(1 \times 1)$ structure of the Te monolayer on the Si surface. The charge density contour plots in Fig. 1(c) indicate that the bond is directional. The maximum of the charge occurs between $\mathrm{Si}$ and Te, but closer to Te. According to Pauling's scale, Te is more electronegative than $\mathrm{Si}, \chi_{\mathrm{Te}}=2.0$ and $\chi_{\mathrm{Si}}=$ 1.8. Therefore, one expects charge to be transferred from $\mathrm{Si}$ to Te. This is consistent with the atomic configurations and occupancies, $\operatorname{Te}\left(5 \mathrm{~s}^{2} 5 \mathrm{p}^{4}\right)$ and $\operatorname{Si}\left(3 s^{2} 3 p^{2}\right)$.

At full monolayer Te coverage, other important issues are the symmetry of the surface and the possible dimerization of the Te adatoms. Given the recent controversy, we attempted to examine this issue thoroughly. Although some experiments have reported missing Te rows and the saturation of the $\operatorname{Si}\left(\begin{array}{lll}0 & 0 & 1\end{array}\right)$ surface at $\Theta=0.8$, there are others [21] that do not find it. In the present work, we ignore any strain-relaxing mechanisms of the missing Te rows and further study the surface reconstruction at 1 ML coverage.

To this end we calculate a free $\mathrm{Te}_{2}$ molecule and find its binding energy to be $4.41 \mathrm{eV}$ and bond length of $2.56 \AA$. The charge density plot for such a $\mathrm{Te}_{2}$ molecule on a plane passing through the $\mathrm{Te}-$ Te bond, shown in Fig. 2(a), reveals the covalent nature of the bond between the two Te atoms. This suggests the possibility that two adjacent Te atoms on the $\operatorname{Si}\left(\begin{array}{ll}0 & 0\end{array}\right)$ surface may experience an energy benefit by forming a Te-Te dimer bond by moving towards each other in the $y$-direction (See 


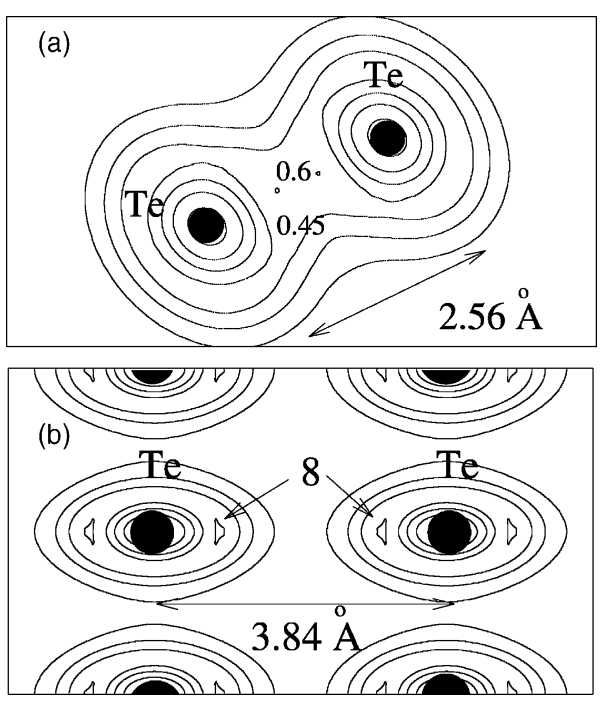

Fig. 2. (a) Charge density contour plots of a $\mathrm{Te}_{2}$ molecule on a plane passing through it. (b) Charge density contour plots on the $\left(\begin{array}{lll}0 & 0 & 1\end{array}\right)$ plane passing through the Te atoms of a monolayer covered $\mathrm{Si}(001)$ surface. Te atoms lie on a square grid of side $3.84 \AA$ A.

Fig. 1(b)). Such a dimerization would lead to a $(1 \times 2)$ reconstruction as proposed by Wiame et al. [21]. As a matter of fact, similar As-As and Al-Al dimerizations have been explored on the As covered $\mathrm{Si}\left(\begin{array}{lll}0 & 0 & 1\end{array}\right)$ and $\mathrm{Ge}\left(\begin{array}{lll}0 & 0 & 1\end{array}\right)$ surfaces $[35,36]$ and $\mathrm{Al}$

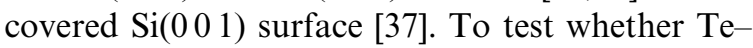
Te dimerization can occur, we examine the following two cases: (i) Starting from an ideal bulk terminated $\operatorname{Si}(001)$ surface, we put Te atoms at the $\mathrm{T}$ and $\mathrm{B}$ sites and relax the system. It should be noted that on the ideal $\mathrm{Si}\left(\begin{array}{lll}0 & 0 & 1\end{array}\right)$ surface the $\mathrm{T}$ and $\mathrm{B}$ and relax the system. It should be noted that on the ideal $\mathrm{Si}\left(\begin{array}{lll}0 & 1\end{array}\right)$ surface the $\mathrm{T}$ and $\mathrm{B}$ sites are equivalent. No forces on the Te atoms were detected in this case and hence Te atoms did not move from these symmetry sites. (ii) An initial structure with a Te-Te distance of $3 \AA$ along $y$ (which is greater than the bond length of $\mathrm{Te}_{2}$, but smaller than the undimerized distance in the $(1 \times 1$ structure) is relaxed at $T=0 \mathrm{~K}$. Upon relaxation, $\mathrm{Te}$ atoms move away from each other, the tilted $\mathrm{Si}-\mathrm{Te}-\mathrm{Si}$ planes become perpendicular to the surface and the total energy of the system is lowered. In both cases, all the Te atoms lie at the same height above the $\operatorname{Si}\left(\begin{array}{ll}0 & 0\end{array}\right)$ surface so that a possible $(1 \times 2)$ surface due to two adjacent Te atoms being at different heights is also ruled out. (The Te-Te dimers proposed by Wiame et al., for example, are reported to be asymmetric in this fashion.) The analysis of the charge density in a (001) (or $x y$ ) plane passing through the Te atoms clearly shows lack of any bonding between them (Fig. 2(b)) since the minimum of the charge density occurs midway between two Te atoms. Formation of strong $\mathrm{Si}-\mathrm{Te}-\mathrm{Si}$ bonds excludes the bonding between two adjacent Te atoms. Simple valence arguments also suggest that $\mathrm{Te}$, being divalent, would tend to avoid bonding with three other atoms. By the same argument, it is not surprising that $\operatorname{As}\left(4 p^{3}\right)$ and $\operatorname{Al}\left(3 s^{2} 3 p^{1}\right)$ form dimers after bonding with two substrate $\mathrm{Si}$ atoms.

An alternate $(1 \times 2)$ structure has been proposed (called the Si-deficit model) apparently based on some features seen in an experiment [38]. In this structure, Te atoms in one row along the $x$ direction, i.e., $\left[\begin{array}{lll}1 & 1 & 0\end{array}\right]$ occupy alternate $\mathrm{T}$ and $\mathrm{B}$ sites (Fig. 1) (on an ideal $\mathrm{Si}(001)$ these sites are equivalent). In the adjacent row, Te atoms simply replace the top-layer $\mathrm{Si}$ atoms, creating a $\mathrm{Si}$ deficit; it has $2 \mathrm{Si}$ atoms less per $(2 \times 2)$ surface cell. Although the resultant symmetry is $(1 \times 2)$, we use a $(2 \times 2)$ computational supercell. The alternate Te rows are located at different heights. The surface unit cell has $2 \mathrm{Si}$ atoms and $4 \mathrm{Te}$ atoms. The "surface" layer can be thought to consist of two atomic planes of Te atoms at dierent heights but the cell symmetry is indeed $(1 \times 2)$. On optimizing this geometry, Te atoms replacing the $\mathrm{Si}$ atoms move along the [1 110$]$ direction by about $0.27 \AA$. Since this structure has $2 \mathrm{Si}$ atoms deficit, we have to take into account this difference in the number of atoms before comparing its energy to the $(1 \times 1)$ $1 \mathrm{ML}$ Te covered $\mathrm{Si}\left(\begin{array}{l}0 \\ 0\end{array}\right)$. To do so, in a system of $(2 \times 2)$ surface cell, we add twice the bulk cohesive energy of $\mathrm{Si}$ (this has to be supplied to remove $2 \mathrm{Si}$ atoms) to the total energy of $(1 \times 1) 1 \mathrm{ML} \mathrm{Te}$ covered $\mathrm{Si}(001)$. This value has to be compared with the total energy of the Si-deficit model plus twice the isolated $\mathrm{Si}$ atom energy. We found the $(1 \times 1)$ structure to be more stable by $0.18 \mathrm{eV}$ per Te adatom, making the Si-deficit model energetically unfavorable. 


\section{Finite temperature MD calculations}

Our calculations have ruled out $(2 \times 1)$ Te covered $\operatorname{Si}\left(\begin{array}{lll}0 & 1\end{array}\right)$ surface for $\Theta=1$ through the formation of Te-Te dimers. We now consider the possibility of such a surface reconstruction due to correlated and concerted motions of the surface and substrate atoms at finite temperatures. Since a zero temperature transition state analysis may not be able to access all the phase space, we perform finite temperature $a b$ initio MD simulations to access additional possible reconstructions. In particular, to verify Tamiya et al.'s claim of a $(2 \times 1)$ surface reconstruction at $T=873 \mathrm{~K}$, we perform MD calculations at $T=600$ and $1000 \mathrm{~K}$ using a $(4 \times 1)$ supercell. Needless to say, a $(4 \times 1)$ supercell cannot give us any large surface reconstructions. However, experiments only report $(1 \times 1)$ and $(2 \times 1)$ surface reconstructions at $\Theta \sim 1$ and a $(4 \times 1)$ cell is sufficiently large for both these possibilities.

At finite temperatures, a definite surface reconstruction would be observed if the Te atoms execute relatively small random thermal motions around positions that are representative of the reconstruction. For example, a possible $(2 \times 1)$ surface would have $\mathrm{Te}$ atoms in a zigzag arrangement. The signature of such a pattern is one in which every alternate atom in a Te chain along $x$ is moved in opposite directions along $y$ (Fig. 1(b)) by the same amount, and oscillates around that point. Another possibility is that alternate atoms in a Te row along $x$ execute oscillations around their ideal $(1 \times 1)$ positions with a phase difference of $\pi$. In the first case, both STM and diffraction experiments would indicate a $(2 \times 1)$ surface. In the second case, an STM experiment will possibly not see a $(2 \times 1)$ image as it will pick up the time-averaged signal. However, diffraction experiments (like LEED) would detect a $(2 \times 1)$ surface as the time scale of interaction of the incident wave with the surface atoms is quite small $(\sim \mathrm{fs})$. In either case, such correlations in the motions of the adatoms would be evident from the paths computed in our MD calculations. If, on the other hand, they execute large uncorrelated motions centered around their ideal $(1 \times 1)$ positions, that would signify the absence of a definite surface reconstruction.

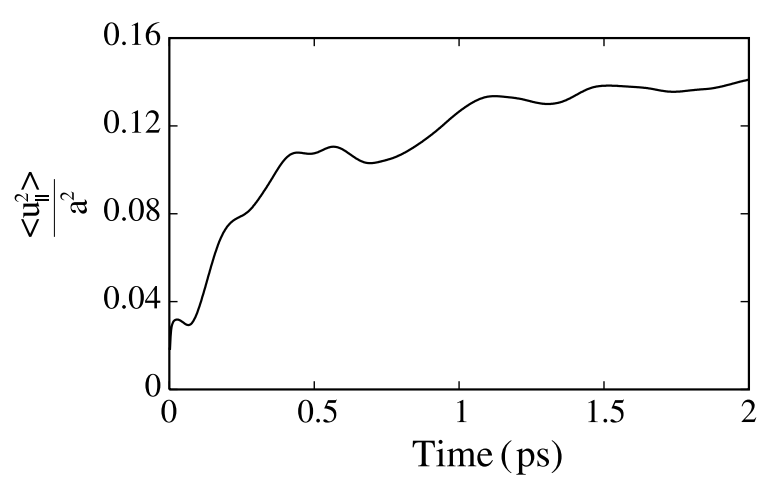

Fig. 3. Time variation of the planar mean square displacements of the Te atoms.

Before examining the paths of the adsorbed Te atoms, we must ensure that the system has thermalized with the thermostat at the specified temperature. For this purpose, we look at the mean squared displacements of the surface atoms in the plane [39]. Fig. 3 shows the quantity

$\frac{\left\langle u_{\|}^{2}(t)\right\rangle}{a^{2}}=\frac{1}{4 t a^{2}} \sum_{i=1}^{4} \int_{0}^{t}\left(u_{x, i}^{2}\left(t^{\prime}\right)+u_{y, i}^{2}\left(t^{\prime}\right)\right) \mathrm{d} t^{\prime}$

as a function of time $t$, where $u_{x}$ and $u_{y}$ are the displacements of the $4 \mathrm{Te}$ atoms from their ideal positions along $x$ and $y$ respectively, and $a$ is the square lattice parameter of the surface. The system

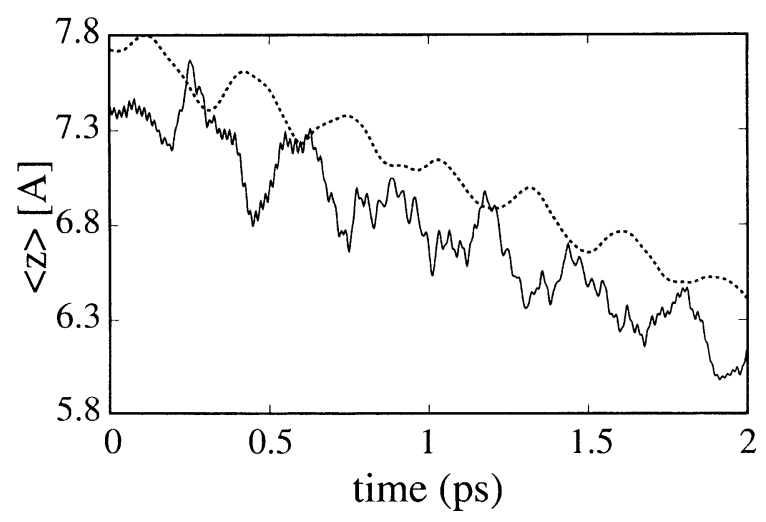

Fig. 4. The mean of $z$-coordinates of atoms in the top (Te, denoted by the dashed line) and bottom layers $(\mathrm{H}$, denoted by the solid line) of the slab as a function of time. The positions of the $\mathrm{H}$ atoms have been shifted vertically by an amount required to show them on the same scale. The average drift velocity $\mathrm{d}\langle z\rangle / \mathrm{d} t \sim-0.7 \AA / \mathrm{ps}$. 
is seen to be sufficiently thermalized within $\sim 1$ ps. The corresponding motion of the surface Te atoms and the bottom layer $\mathrm{H}$ atoms along $z$ are shown in Fig. 4. Due to its finite size, the whole system acquires a spurious velocity along the $z$-direction whose magnitude is $\mathrm{d}\langle z\rangle / \mathrm{d} t \sim 0.7 \mathrm{~A} / \mathrm{ps}$.

Fig. 5 illustrates the displacements of the Te atoms along $x$ and $z$ in a $(4 \times 1)$ supercell at $T=600 \mathrm{~K}$. The displacements along the $x$-direction, $u_{x, i}(t)$, are small, the maximum displacement being $\sim 0.3 \AA$. This is because the displacements along $x$ involve stretching and compressing Te-Si bonds, which are not favorable energetically. A plot of $u_{z, i}(t)$ for all the Te atoms show that, in addition to the spurious translation of the unit cell, the displacement of each Te atom, $u_{z, i}(t)$, oscillates with decreasing amplitude and without any correlation with the other Te atoms. This rules out the hypothesis of a $(2 \times 1)$ pattern originating from alternate Te at different heights.

The displacement along the [1 10$]$ (or $y$ ) direction, $u_{y, i}(t)$, is large and can be relevant for a zigzag reconstruction. After the thermalization of the system, $u_{y, i}(t)$ becomes oscillatory and quasi periodic with periods of the order of $\sim 1.0$ ps. The displacements of the Te atoms along $y$ at $T=600$ and $1000 \mathrm{~K}$ are shown in Fig. 6. This is reminiscent of the surface longitudinal acoustic mode due to Te rows. The amplitudes of the oscillations vary between 0.4 and $0.7 \AA$ resulting in lateral excursions as large as $1.4 \AA$ of Te rows along the [1 10$]$ direction at $T=600 \mathrm{~K}$. As expected, the ampli-
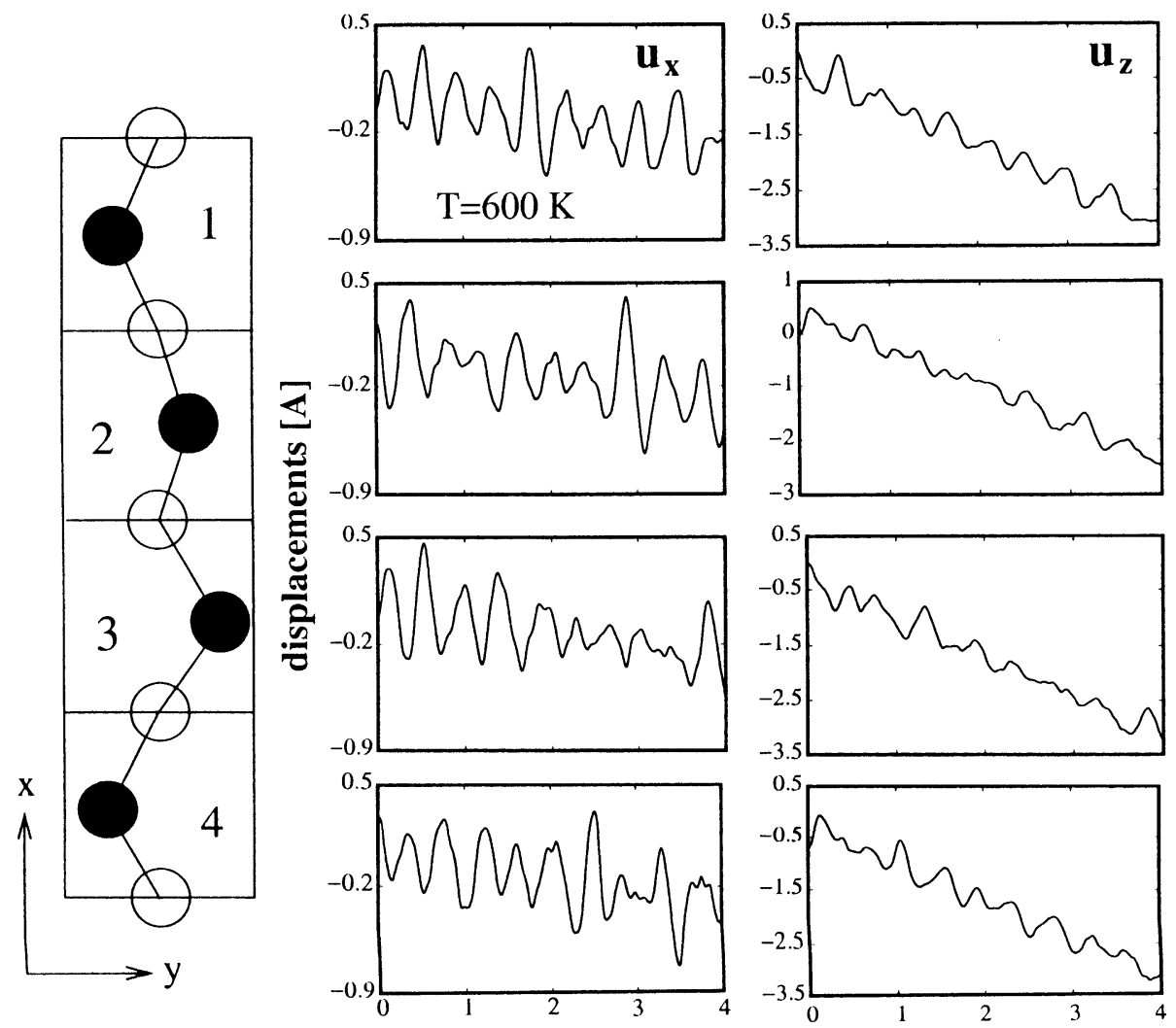

\section{time (ps)}

Fig. 5. Time variation of the displacements, $u_{x, i}$ and $u_{z, i}$, of Te atoms $(i=1, \ldots, 4)$ in a $(4 \times 1)$ supercell. Ab initio MD calculations were performed at $T=600 \mathrm{~K}$. A $(4 \times 1)$ supercell, rotated by $90^{\circ}$ (note the horizontal axis is $y$ and vertical axis is $x$ ) is shown in the left panel for ease of comparison with the displacements of Te atoms. 

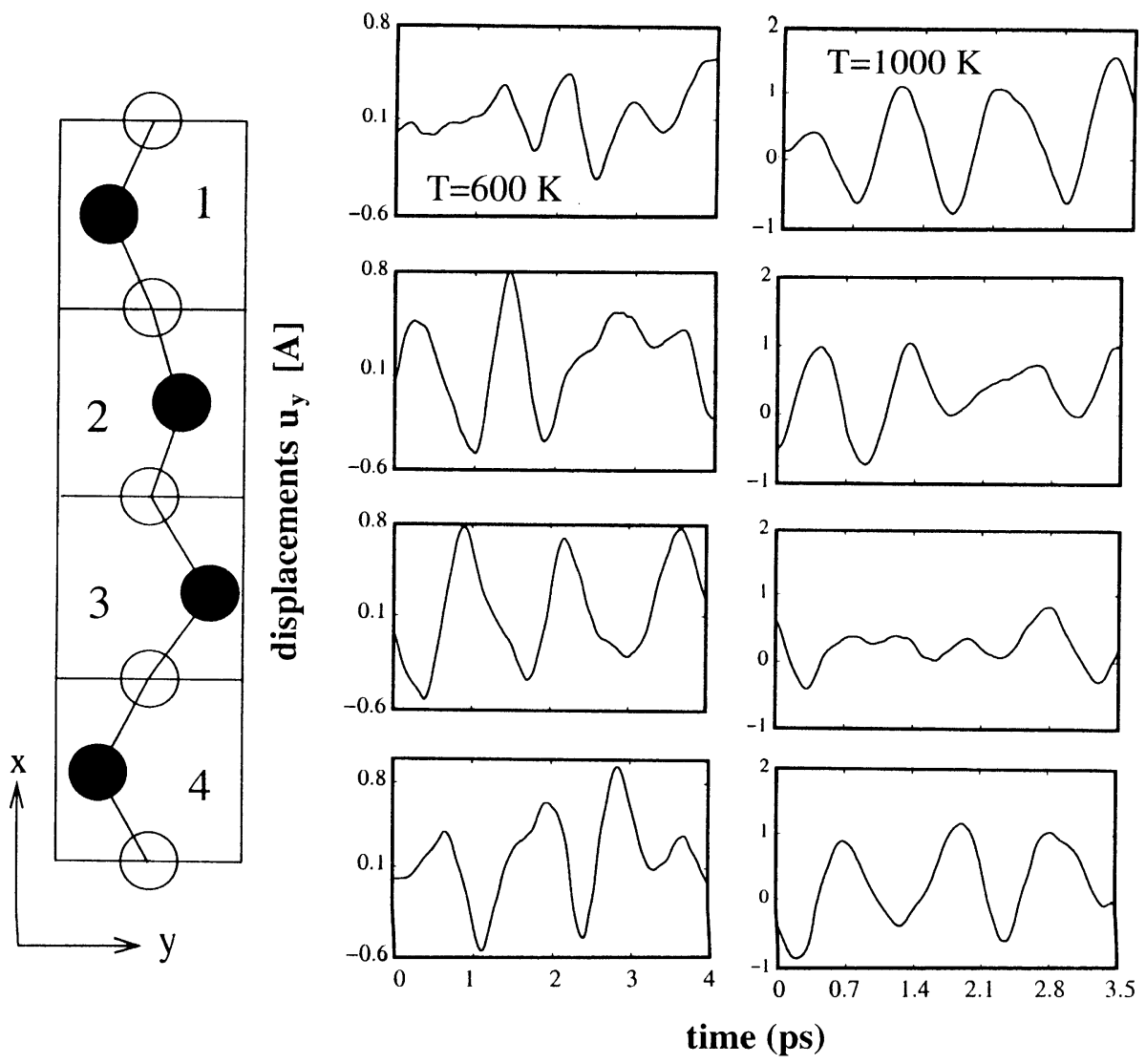

Fig. 6. Time variation of the displacement of atoms, $u_{y, i}$, along the $[1 \overline{1} 0]$ direction calculated at $T=600$ and $1000 \mathrm{~K}$.

tudes are larger at $T=1000 \mathrm{~K}$. In this case the excursion can be as large as $\sim 2 \AA$. (See for example, Te atom 4 in Fig. 6.) Such unusually large lateral excursions of the Te adatoms explain the small coherent fraction observed in the XSW spectroscopy experiments on this surface [40]. As is evident in Fig. 6, displacements of adjacent rows do not display any correlation. The random and uncorrelated nature of the displacements prevents the development of any well-defined reconstruction pattern.

Adsorbed $\mathrm{Te}$ rows executing large amplitude excursions with low frequency along the $y$-direction at finite temperatures is unusual and suggests rather soft and non-Hookian (non-linear) force constants in this direction. This is supported by the total energy curves shown in Fig. 7. The total energy remains practically unchanged for a dis- placement of the Te rows of $u_{y} \sim \pm 0.5 \AA$. For the displacement of adjacent rows in opposite directions, $E_{\mathrm{T}}\left(u_{y}\right)$ is like a double well potential with a broad maximum at $u_{y}=0$ and a shallow minimum on both sides. The barrier between these two minima is very small and is at the accuracy limit of the present calculations $(7 \mathrm{meV}$ for a $(2 \times 1)$ supercell).

Interestingly, except for the disappearance of the weak double well form, the variation of the total energy with $u_{y}$ remains essentially unaltered, if the adjacent Te rows are displaced in the same direction. This implies that, at finite temperatures, Te rows can easily go over the weak barrier. This is precisely the reason we see the Te atoms executing random (uncorrelated) motions along $y$ in our finite temperatures MD calculations shown in Fig. 6. The total energy curves in Fig. 7 are fit to an 


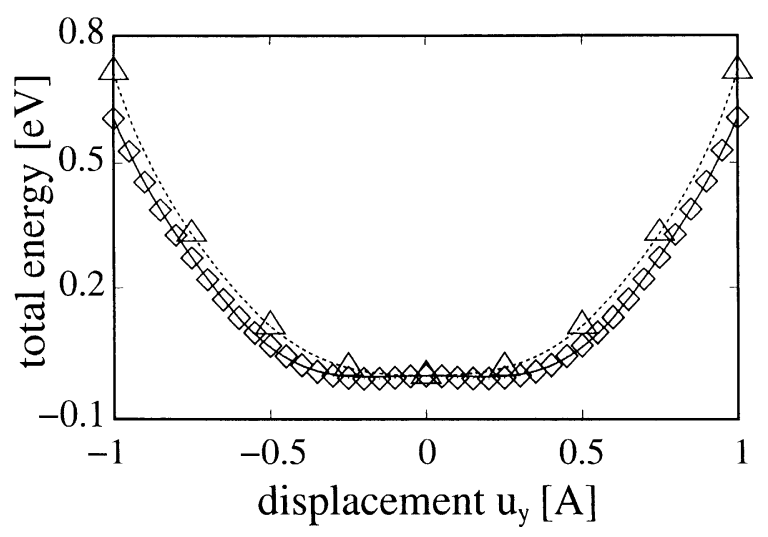

Fig. 7. Variation of the total energy with the displacement of the Te row, $u_{y}$, calculated at $T=0 \mathrm{~K}$. Each data point corresponds to a fully relaxed structure under a given displacement of the Te rows along the [1 110$]$ direction. The diamonds $(\diamond)$ are for the adjacent rows moving in opposite directions forming a zigzag pattern and the $\Delta$ 's are for the Te rows moving in the same direction. The dashed and solid lines are fits to the diamonds and the $\triangle$ 's respectively, as discussed in the text.

analytical form $E_{\mathrm{T}}\left(u_{y}\right)=\alpha u_{y}^{2}+\beta u_{y}^{4}+\gamma u_{y}^{6}+\delta u_{y}^{8}$. For displacements of all the Te atoms in the same direction, the total energy can be fit with $\alpha=$ $0.2128 \mathrm{eV} / \AA^{2}, \quad \beta=1.2976 \mathrm{eV} / \AA^{4}, \quad \gamma=-1.5531$ $\mathrm{eV} / \AA^{6}$, and $\delta=0.7607 \mathrm{eV} / \AA^{8}$. On the other hand, the total energy for zigzag displacements of the Te atoms can be fit with $\alpha=-0.1379 \mathrm{eV} / \AA^{2}, \beta=$ $2.0978 \mathrm{eV} / \AA^{4}, \gamma=-2.2809 \mathrm{eV} / \AA^{6}$, and $\delta=0.9313$ $\mathrm{eV} / \AA^{8}$. In both these cases, strong anharmonicity of the potential wells, wherein the Te atoms move, are clear.

It is instructive to examine the band structure of $\mathrm{a}(1 \times 1)$ and $(2 \times 1)$ Te-covered surface due to zigzag arrangement of the adatoms. We have plotted in Fig. 8(a) the bands for $(1 \times 1)$ structure in a $(2 \times 1)$ unit cell, so as to compare the folded bands with the actually computed bands for the $(2 \times 1)$ structure. The only obvious effect of the full self-consistency is to slightly shift the bands away from the Fermi level. Although these shifts are at the limit of accuracy of our calculations, there is a hint that the band gap at $\Gamma$ increases with respect to the $(1 \times 1)$ structure.

Large and random excursions of Te rows along the $[110]$ direction would not give rise to any resolvable pattern in the LEED and STM images. For example, since the period of oscillations are

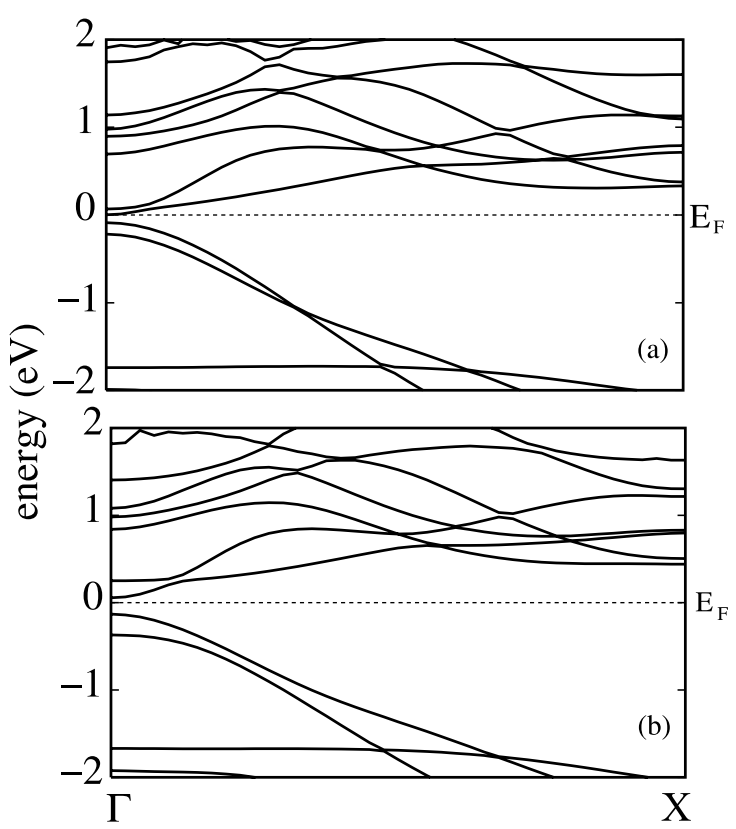

Fig. 8. (a) Band structure of $(1 \times 1)$ monolayer of Te covered

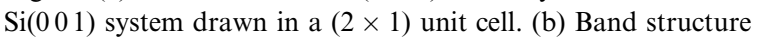
when the Te rows are displaced in a zigzag manner by $0.25 \AA$.

much shorter than the characteristic scan time of STM, the STM images taken at finite temperature would indicate disordered $(1 \times 1)$ reconstruction. Moreover, since the potential energy well is so flat, positions of the Te atoms could be easily modified by the tip-sample interaction in STM experiments. This leads to additional complications in deducing a surface reconstruction pattern from STM data. We have also seen that the alternate $(2 \times 1)$ structure proposed is a higher energy one compared to a $(1 \times 1)$ structure. In view of this, it seems that there are two possibilities as to why some experiments see $(2 \times 1)$ surface reconstruction. First, the surface atoms getting trapped in some metastable state during the formation of the Te adlayer. Second, the only major way in which the experiments of Wiame et al. and others differ is that in Wiame's experiments, Te is deposited at elevated temperatures (between 500 and $850{ }^{\circ} \mathrm{C}$ ), while the corresponding temperatures in all the other experiments are relatively low (room temperature to $200{ }^{\circ} \mathrm{C}$ ). All the measurements are, however, done at room temperature. While cooling the system to room temperature from $500{ }^{\circ} \mathrm{C}$, 
it is possible that because of their increased mobility at high temperatures, the adatoms and substrate can explore lower energy configurations which are difficult to reach starting from a particular low-temperature configuration.

\section{Summary}

In summary, we have found that Te atoms

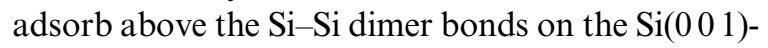
$(2 \times 1)$ surface at low coverage. At a half monolayer coverage, the $\mathrm{Si}-\mathrm{Si}$ dimers on the surface survive, though they are weakened slightly and their asymmetry is removed. There is no energy benefit for forming Te dimers at any coverage. At monolayer coverage, the potential well for $\mathrm{Te}$ atoms is rather flat and strongly anharmonic along the [1 10$]$ direction. There is almost no barrier for the Te rows on the surface to make significant excursions relative to their ideal positions along this direction. $\mathrm{Ab}$ initio finite temperature MD calculations indicate that the displacements of Te rows are uncorrelated lacking any definitive reconstruction pattern.

\section{Acknowledgements}

We acknowledge support for this work from the Army Research Laboratory (ARL) through the Collaborative Technology Alliance (CTA) program. One of us (IPB) is also grateful to UIC campus research board for the seed grant which enabled us to initiate this research project in the Physics Department.

\section{References}

[1] R.I.G. Uhrberg, R.D. Bringas, R.Z. Bachrach, J.E. Northrup, Phys. Rev. Lett. 56 (1986) 520.

[2] R.D. Bringas, R.I.G. Uhrberg, M.A. Olmstead, R.Z. Bachrach, Phys. Rev. B 34 (1986) 7447.

[3] D.J. Eagelsham, M. Cerullo, Appl. Phys. Lett. 58 (1991) 2276.

[4] H.J. Osten, J. Klatt, G. Lippert, E. Bugiel, S. Hinrich, Appl. Phys. Lett. 60 (1992) 2252.

[5] G. Meyer, B. Voitlander, N.M. Amer, Surf. Sci. 274 (1992) 1541.
[6] S. Higuchi, Y. Nakanishi, Surf. Sci. 254 (1991) L465.

[7] H.J. Osten, J. Klatt, G. Lippert, E. Bugiel, S. Higuchi, J. Appl. Phys. 74 (1993) 2507.

[8] M.R. Bennett, C.J. Dunscombe, A.A. Cafolla, J.W. Cairns, J.E. Macdonald, R.H. Williams, Surf. Sci. 380 (1997) 178.

[9] Y.W. Mo, B.S. Swartzentruber, R. Kariotis, M.B. Webb, M.L. Lagally, Phys. Rev. Lett. 63 (1989) 2392.

[10] Y.W. Mo, J. Vac. Sci. Technol. A 8 (1990) 201.

[11] Y.W. Mo, M.G. Lagally, Surf. Sci. 248 (1991) 313.

[12] D. Dijkkamp, E.J. van Loenen, H.B. Elswijk, in: Proceedings of the Third NEC Symposium on Fundamental Approach to New Material Phases, Springer Series of Materials Science, Springer, Berlin, 1992.

[13] A.P. Smith, H. Jonsson, Phys. Rev. Lett. 77 (1996) 1326.

[14] C.D. Consorte, C.Y. Fong, M.D. Watson, L.H. Yang, S. Ciraci, Phys. Rev. B 63 (2001) R041301-1.

[15] M.R. Bennett, A.A. Cafolla, J.W. Cairns, C.J. Dunscombe, R.H. Williams, Surf. Sci. 360 (1996) 187.

[16] A. Almeida, Y.P. Chen, J.P. Faurie, S. Sivananthan, D.J. Smith, S.C. Tsen, J. Electron. Mater. 25 (1996) 1402.

[17] S.A. Yoshikawa, J. Nogami, C.F. Quate, P. Pianetta, Surf. Sci. 321 (1994) L183.

[18] S. Di Nardo, L. Lozzi, M. Passacantando, P. Picozzi, S. Santucci, Surf. Sci. 331-333 (1995) 569.

[19] S. Santucci, L. Lozzi, M. Passacantando, P. Picozzi, Surf. Sci. 351-354 (1996) 1027.

[20] S.R. Burgess, B.C.C. Cowie, S.P. Wilks, P.R. Dunstan, C.J. Dunscombe, R.H. Williams, Appl. Surf. Sci. 104-105 (1996) 152.

[21] F. Wiame, G. Mahot, S. Sivananthan, S. Rujirawat, R. Caudano, R. Sporken, Appl. Surf. Sci. 142 (1999) 475.

[22] N. Grandjean, J. Massies, Phys. Rev. B 53 (1996) R23231.

[23] S. Gundel, W. Faschinger, Phys. Rev. B 59 (1999) 5602.

[24] K. Tamiya, T. Ohtani, Y. Takeda, T. Urano, S. Hongo, Surf. Sci. 408 (1998).

[25] R.H. Miwa, A.C. Ferraz, Surf. Sci. 449 (2000) 180.

[26] N. Takeuchi, Phys. Rev. B 60 (1999) 4796.

[27] P. Sen, S. Ciraci, I.P. Batra, C.H. Grein, Phys. Rev. B 64 (2001) 193310.

[28] H.J. Monkhorst, J.D. Pack, Phys. Rev. B 13 (1976) 5188.

[29] D. Vanderbilt, Phys. Rev. B 41 (1990) 7892; G. Kresse, J. Hafner, J. Phys.: Condens. Matter 6 (1994) 8245.

[30] J.P. Perdew, Y. Wang, Phys. Rev. B 46 (1992) 6671.

[31] S. Nosé, J. Chem. Phys. 81 (1984) 511.

[32] G. Kresse, J. Hafner, Phys. Rev. B 47 (1993) R558.

[33] G. Kresse, J. Furtmüller, Phys. Rev. B 54 (1996) 11169.

[34] A. Ramstad, G. Brocks, P.J. Kelly, Phys. Rev. B 51 (1995) 14504.

[35] M. Tromp, M.C. Reuter, Phys. Rev. Lett. 68 (1992) 954.

[36] R.I.G. Uhrberg, R.D. Bringas, R.Z. Bachrach, J.E. Northrup, Phys. Rev. Lett. 56 (1986) 520.

[37] I.P. Batra, Phys. Rev. Lett. 65 (1989) 1704.

[38] R. Sporken, Private communication.

[39] N. Marzari, D. Vanderbilt, A. De Vita, M.C. Payne, Phys. Rev. Lett. 82 (1999) 3296.

[40] D. Walko et al., Preprint. 